Relations industrielles

Industrial Relations

\title{
Emploi ou assistance, par Bent Anderson, Organisation de Coopération et de Développement Économique, Paris 1966. 125 pages.
}

\section{Michel Bergeron}

Volume 22, numéro 2, 1967

URI : https://id.erudit.org/iderudit/027801ar

DOI : https://doi.org/10.7202/027801ar

Aller au sommaire du numéro

Éditeur(s)

Département des relations industrielles de l'Université Laval

ISSN

0034-379X (imprimé)

1703-8138 (numérique)

Découvrir la revue

Citer ce compte rendu

Bergeron, M. (1967). Compte rendu de [Emploi ou assistance, par Bent Anderson, Organisation de Coopération et de Développement Économique, Paris 1966. 125 pages.] Relations industrielles / Industrial Relations, 22(2), 307-307. https://doi.org/10.7202/027801ar

Tous droits réservés @ Département des relations industrielles de l'Université Laval, 1967
Ce document est protégé par la loi sur le droit d'auteur. L’utilisation des services d'Érudit (y compris la reproduction) est assujettie à sa politique d'utilisation que vous pouvez consulter en ligne.

https://apropos.erudit.org/fr/usagers/politique-dutilisation/ 
lection of Trainees Under MDTA ». En effet, il est loisible de penser que la motivation des travailleurs est justement un critère très important en ce qui a trait à lo sélection des travailleurs eu égard aux cours de formation professionnelle.

Plus spécifiquement, ce présent rapport tente d'expérimenter une mesure et plus particulièrement un ensemble de mesures qui devraient permettre aux enquêteurs d'en arriver à une connaissance plus systématique de la motivation ou travail chez la masse des travailleurs.

D'une façon assez synthétique, le rapport veut au départ familiariser le lecteur avec les composantes théoriques de la motivation. $A$ ce sujet, on y dit que la motivation individuelle vis-à-vis un objectif à atteindre peut être comprise de trois façons: d'abord la motivation face au but à atteindre, l'espoir de l'atteindre qui se mesure dans le degré d'attente ou d'expectative et enfin la valeur du stimulus que comporte cet objectif.

D'une façon plus concrète, le rapport élabore ensuite une série de méthodes qui se doivent de mesurer le plus fidèlement possible la motivation, la validité de la mesure de la motivation, l'attente et le stimulus. Enfin, en dernière analyse, les auteurs du rapport ont voulu confronter les résultats obtenus avec le comportement potentiel des travailleurs à leur travail et ils en concluent $q^{\prime}$ il existe une relation statistique entre ce même comportement et les variables qui sont reliées à la motivation au travail.

En somme, les auteurs ont voulu développer des mesures, des techniques et des méthodes susceptibles d'analyser la motivation ou travail. D'où il est maintenant possible d'identifier l'impact des divers cours de formation professionnelle sur la motivation individuelle au travail. II en ressort également qu'il peut arriver que les caractéristiques motivationnelles qui peuvent faciliter l'adaptation du travailleur à son travail ne sont pos nécessairement celles qui facilitent la réussite dans un cours de formation professionnelle. De toute façon, cette restriction n'altère en rien la validité et l'intégrité de l'étude; au contraire, il s'ensuit logiquement que la voie est maintenant toute tracée en vue de l'approfondissement des résultats obtenus et de la découverte de nouvelles possibilités. II n'y a donc pas lieu de désespérer, le champ presqu'inextricable et invulnérable des relations humaines, dans le plein sens du mot, se laisse de plus en plus opprivoiser par les chercheurs.

Paul BOUCHARD
Emploi ou assistance, par Bent Anderson, Organisation de Coopérotion et de Développement Economique, Paris 1966. 125 pages.

Chacun connait l'importance de plus en plus grande qui est donnée oux programmes de sécurité sociale. II y a d'autre part I'apparition de concepts économiques et sociaux préconisant la participation active de tous les groupes à l'effort de production d'un pays. Ce rapport de I'O.C.D.E. traite justement des problèmes d'emploi des personnes qui, ò un moment donné, sont difficiles à placer.

Emploi ou assistance constitue une analyse des coûts et bénéfices, ainsi qu'une évaluation des gains escomptables du point de vue social et humain de l'emploi des personnes dites de «groupes spécicux .

Un emploi comporte habituellement des exigences précises: santé, âge, etc. Et $M$. Anderson se presse d'ajouter que certaines personnes sont repoussées du marché du travail à la fois par des caractéristiques personnelles et des conditions socio-économiques. Que faire pour ces gens? Assistance ou emploi. Et l'auteur préconise de prendre des mesures en vue de créer des possibilités d'emploi compensant l'inaptitude à souteniir la concurrence pour les emplois normaux, ou divers moyens propres à assurer un emploi permanent de remplacement. Point n'est besoin d'insister sur la complexité de dégager les solutions.

Toutefois l'auteur présente au chapitre IV les résultats d'une étude pilote: étude faite par questionnaire dans six pays: le Danemark, les Pays-Bas, la République Fédérale d'Allemagne, le Royaume-Uni, la Suède et les Etats-Unis. L'auteur avoue ne pouvoir se faire une idée nette et précise des bénéfices et des coûts de l'emploi des a groupes spéciaux », mais peut cependant voir les rapports multiples du problème.

Ce bref rapport demeure facile d'accès, et se termine sur des considérations de projets futurs de recherche. Mais I'utilité du présent rapport serait indiscutable vu les problèmes semblables que nous connoissons ici ou Québec.

\section{Michel BERGERON}

Manpower Research Projects, Sponsored by the U.S. Department of Labor, Manpower Administration, Washington, D.C., 20210, 1966, 129 pages. 